\title{
Practice Note: Menstrual Health Management in Humanitarian Settings
}

\author{
Marianne Tellier, Alex Farley, Andisheh Jahangir, \\ Shamirah Nakalema, Diana Nalunga, and Siri Tellier
}

\section{INTRODUCTION $^{1}$}

The 2015 earthquake in Nepal affected an estimated 1.4 million women of reproductive age (Chaudhary et al. 2017, 1). Women ranked the need for menstrual hygiene products as a top priority (after food, but ahead of soap and medicine). However, none reported receiving such products during the first month after the earthquake, while more than $50 \%$ reported receiving soap (Budhathoki et al. 2018, 3). Meanwhile, in Uganda, about 1 million refugees have lived in refugee settlements for years or even decades, and rely on food rations for daily sustenance. But a local trainer reports that women have difficulties walking to distant food distribution points while menstruating, as they chafe and stain. Some women sell their food rations to buy pads (CARE International and WoMena Uganda 2018, 9).

We are volunteers or staff with WoMena, an NGO which has worked since 2012 to improve menstrual health and management in over 100 settings in Ugandan communities, including in refugee settlements. This review reflects on the challenges associated with meeting menstrual health needs in various humanitarian settings through an analysis of the 2015 Nepal earthquake, a short-term emergency setting, and some of our findings to date from the long-term settings of Kitgum, Rhino, Bidibidi, and Imvepi. 
While the term 'menstrual hygiene management' has long been widely used (WHO and UNICEF 2012, 14), we refer to 'menstrual health and management' since menstruation is not only an issue of 'hygienic' management of something 'dirty', but should be expanded to the concepts of wider physical, mental, and social well-being, as conceptualized in the WHO Constitution (WHO 1946, 1). We use the term 'menstruators' in recognition that not all who menstruate are girls or women, and not all who are girls or women menstruate. Finally, we use the term 'humanitarian settings' to refer to situations where a major 'human-made' or 'natural' disaster has disrupted the livelihoods and/or support systems of a community to an extent that the community cannot cope without outside assistance (Tellier et al. 2017, 120). The affected population may be dependent on outside organizations for basic needs such as food and housing.

Disasters affect millions of people every year. They may result in forced displacement, and the UNHCR estimated there were over 25 million refugees and 40 million internally displaced persons in 2017 (UNHCR 2018b, 2 ). Humanitarian settings vary widely. One misconception is that disasters develop suddenly and revert to a more 'normal' state within months. In fact, many last for decades. UNHCR estimates as of 2015 showed that the average duration of 32 protracted refugee situations around the globe was 26 years (UNHCR 2016, 20). Evidence related to health in humanitarian settings is generally poor (Blanchet et al. 2017, 1). The topic of menstrual health is no exception, but the following summarizes some main findings from the limited literature that is available.

\section{Menstrual Health Challenges in Humanitarian Settings}

Menstruators living in humanitarian settings face many of the same challenges as those in more stable situations, but may find that their challenges are exacerbated. Barriers to menstrual health are numerous, complex, and interconnected, and one person's lived experiences with menstruation cannot be used to fully describe the experiences of all other menstruators. Therefore, we have attempted to structure the following section according to simple, overarching themes which tend to underpin menstrual health management.

\section{Menstrual Shame and Stigma}

Stigma is at the root of many challenges related to menstrual health, and often manifests in menstruators experiencing shame and embarrassment (Johnston-Robledo et al. 2013, 1). In refugee settings, common fears of chafing, staining, and odor are exacerbated, for instance, when menstruators walk long distances to food distribution points (CARE International and WoMena Uganda 2018, 11). Such discomforts may induce stress: $60 \%$ of Syrian refugees reported stress due to lack of underwear (Pujol-Mazzini 2017, 1), refugees in Iran reported that asking unfamiliar male health providers for help 
caused stress (Sohrabizadeh et al. 2018,4) and, for refugees in Jordan, drying materials where unfamiliar males may see them is a major problem (ACF et al. 2017, 4-12).

In essence, the negative impacts of stigma bleed into menstruators' daily lives.

\section{Health, Including Sexual and Reproductive Health and Rights (SRHR)}

Circumstances typical to humanitarian settings and the general stigma and lack of information that surround menstruation often converge to act as barriers to the physical and mental health of menstruators. Menstrual stigma prevents many women from seeking medical attention from aid agencies (Pujol-Mazzini 2017), and the stress associated with menstrual disorders may be prompted or exacerbated by the volatility of emergency settings (VanLeeuwen and Torondel 2018, 356). Such restrictions on menstruators' comfort and ability to access health services prevents them from receiving diagnoses and treatment for health issues, and cuts them off from streams of information on health and hygiene, menstrual or otherwise.

Urogenital infections have a high prevalence (20-30\% or more), in high-, middle-, and low-income countries. However, whereas it seems plausible that there is a connection between different materials or practices, there is as yet little evidence, either in humanitarian or more stable settings (Balls et al. 2017, 1; WoMena 2019a, 1). Some studies are beginning to shed some light on this, but further studies are needed to draw general conclusions (Torondel et al. 2018, 8-9; Phillips-Howard et al. 2016, 4-5).

Limitations on resources and economic opportunities in emergency settings may also compromise health. Menstruators may take recourse to the strategy of engaging in sex work to pay for basic needs, including menstrual materials (Phillips-Howard et al. 2016, 1). Such strategies place menstruators at higher risk for gender-based violence and contracting sexually transmitted infections (Women's Refugee Commission 2016, 5, 7), and are reported to increase in humanitarian settings where the accessibility of menstrual materials is poor (Samari 2017, 256).

\section{Access to Menstrual Materials, Including Affordability and Acceptability}

Affordability of menstrual materials is a widespread problem, sometimes referred to as 'period poverty.' That problem may be exacerbated in refugee contexts, where many people have lost their livelihood. Humanitarian organizations often hand out menstrual materials in kits, which are intended to last for three months. The cost to humanitarian agencies is high: UNFPA kits, for example, cost on average 22 USD (Abbott et al. 2011, 44). Some organizations continue with distributions for decades, but standards dictating frequency and overall duration of distribution programs vary greatly. Many organizations do so only for the first three months of an emergency 
(Sommer 2012, 91; Kragelund Nielsen 2017, 11) with the expectation that, when they stop distributions, menstruators will revert to customary sources, whatever they may be.

Apart from affordability, determining which products are 'acceptable' presents a major challenge to aid workers. Some authors assume that acceptability can be inferred from 'usual practice' before the emergency, but usual practices may be difficult to identify and implement (Parker et al. 2014, 449$50)$. The Sphere minimum standards ${ }^{2}$ recommend that assistance for a long list of activities, including the planning and provision of "menstrual hygiene supplies and facilities" and waste disposal mechanisms (Sphere Association $2018,100,102$ ), should be culturally and contextually appropriate. However, it provides little guidance on how to achieve this, given that the text is a set of minimum standards, rather than guidelines (Sphere Association 2018, 104). Community consultations on the cultural and contextual nuances of local menstrual practices are likely to cause programming delays in the early phases of an emergency (Abbott et al. 2011, 59), and are therefore typically carried out in later phases, when menstrual materials may no longer be distributed.

Without contextual insight from consultations, humanitarian organizations may distribute products that are unfamiliar to menstruators or which may be unsuitable for the setting. For instance, recipients of disposable pads after the Pakistan earthquake used them for various household purposes (Sommer et al. 2016, 255). Older menstruators may see reusable pads as a sustainable option, whereas adolescent girls may find it difficult to manage washing and drying (Robinson and Obrecht 2016, 17; Kågesten et al. 2017, 15). Moreover, culture is a fluid concept, but there is little literature focusing on the trends and influencing factors behind changes in menstruators' views over time. One humanitarian staffer notes that refugee women are eager to try new products and that, "they are all the time on the phones and they watch Instagram, Facebook, and YouTube, and they do have advertisement about the cup, for example, and they ask me what it is" (VanLeeuwen and Torondel $2018,355)$. In addition to these complexities in obtaining acceptable menstrual materials, menstruators in refugee settings also typically struggle to find the safe and adequate spaces needed for changing, washing, or disposing of said materials, as well as for carrying out other menstrual practices.

\section{Water, Sanitation, and Hygiene, Including Environmental Concerns}

Sanitation facilities in camps are often inadequate for menstruators: they are neither sex segregated, adequately private nor sufficiently lighted (Atuyambe et al. 2011, 1). Obtaining water for washing materials and soiled garments is a problem, as is finding inconspicuous spaces for drying. Menstruators may dispose of materials in toilets or latrines instead of bins because they fear being observed (Elledge et al. 2018, 9). 


\section{Populations with Particular Needs}

The cycle of issues that feed and characterize gaps in the menstrual health of refugees is amplified for populations with particular needs. Menstruators with disabilities and their caretakers are likely to have special, unmet needs (Myers et al. 2018, 7; Rasanathan et al., forthcoming). Unaccompanied or orphaned girls may also face unique challenges, as they often lack a social support network. Moreover, existing discrimination based on indigenous or minority status may be exacerbated in humanitarian emergencies (Sommer, Schmitt, and Clatworthy 2017, 63). From the margins of their communities, these groups encounter unique and profound difficulties accessing the benefits of international and local relief efforts.

\section{Response AT THE INTERNATIONAL LEVEL}

Following a crisis, a government's capacity to lead and coordinate relief efforts within its borders may be temporarily compromised. The chaos means not only that pre-existing problems of the affected populations are multiplied, but also that government capacities to meet their populations' needs are decimated: staff fatalities soar, and buildings, computer systems, and electric supplies are demolished. A wide range of international actors may rapidly begin operations to fill infrastructural gaps, therefore at such times, international coordination becomes key. In 1991, a UN resolution was adopted to step up and improve coordination in international humanitarian action (UN General Assembly 1992, 1). Subsequently, an extensive set of structures, policies, and guidelines has been developed by UN organizations, while the aforementioned Sphere Standards have been developed by the Red Cross/Red Crescent and over 600 humanitarian practitioners representing non-governmental organizations of various sizes from over 80 countries.

Water, sanitation, and hygiene received early attention in international coordination efforts, and the sector was one of the first to identify menstruation as an issue (Sommer 2012, 84). Menstruation would seem to be a good fit for the reproductive health field. However, it took time before reproductive health in itself was recognized as a component of humanitarian assistance, since it was not perceived as life-saving (Chynoweth 2015, 2 ). It took even longer before menstruation was perceived as part of overall reproductive health. One of the first acknowledgements was in 2000, when the UNFPA recommended that the response to reproductive health should include 'dignity kits' with menstrual materials, and successfully advocated that such services are life-saving and therefore eligible for humanitarian funding (Abbott et al. 2011, 14, 16).

Gradually, the scope of menstrual health response was widened beyond materials. In 2016 Sommer et al. proposed that such response needs to entail the, "provision of safe, private, and hygienic water and sanitation facilities for changing menstrual materials and bathing, easy access to water inside or near 
toilets, supplies (e.g. laundry soap, separate basin) for washing and drying menstrual materials discreetly, disposal systems through waste management, and access to practical information on MHM (menstrual hygiene management), for adolescent girls in particular" (Sommer et al. 2016, 247). The 2018 version of the Sphere Standards includes guidance on the provision of menstrual health 'hardware,' such as the suggestion of providing 15 disposable pads per month or of providing a bin and incinerator for waste; and it features guidance on providing software, such as information about menstruation for students and the consultation of women on their preferred menstrual health practices (Sphere Association 2018, 104). In 2017, Columbia University and the International Rescue Committee published a toolkit for integrating Menstrual Hygiene Management (MHM) into humanitarian response (Sommer et al. 2017). Their work also helped to build recognition that higher level buy-in was necessary for tangible changes in menstrual health programming to take place. In 2018, the UNFPA convened a symposium calling for more attention to menstruation in general within reproductive health, and specifically in humanitarian settings, with a focus on longer-term sustainability in regards to policy and financing (Department of Women, South Africa, and UNFPA 2018, 6).

Thus, much progress has been made at the international level. However, "[d]espite a growing dialogue around MHM (menstrual hygiene management) in emergencies, there remains a lack of clarity on the key components for a complete MHM response, the responsible sectoral actors to implement MHM activities, the most effective interventions to adapt in emergency contexts, and insufficient guidance on monitoring and evaluation" (VanLeeuwen and Torondel et al. 2018, 1). One prominent contributor to the lack of global consensus in these areas is the fact that beneficiary needs and the determinants of 'appropriate' response can vary greatly from one national context to another.

\section{Response at the National Level}

To provide an indication of the great variation in humanitarian settings, we list some lessons learned from two different contexts: the 2015 earthquake in Nepal and long-term refugee settlements in Uganda.

\section{Nepal}

In the aftermath of the earthquake, organizations found it challenging to provide all menstruators, estimated at around 1.4 million, with menstrual materials (seemingly mostly disposable pads) (Chaudhary et al. 2017, 37). One issue was affordability. In order to have provided all menstruators with disposable pads according to the Sphere minimum standards, 750,000 pads would have to have been financed, produced, distributed, and disposed of, per day. In reality, materials were unevenly provided, and there was little 
attention given to issues beyond provision, such as the logistics of disposal (Myers et al. 2018, 2-9). The Nepal Red Cross Society imported a machine from India for making affordable disposable pads. However, the machine was found to require high maintenance, and the cost (around 0.05 USD per pad) was not sufficiently low to be affordable (Bharati, email to Siri Tellier, 13 April 2018). Some authors suggest that reusable cloth was popular both pre- and post-earthquake, and should also be used in emergencies instead of introducing disposable pads (Budhathoki et al. 2018, 6). One response implemented in the 14 most affected districts included teaching adolescents to make reusable pads using old clothes. Beyond the focus on materials, the program trained school teachers and health workers on how to educate adolescents about menstruation, and on providing adolescent-friendly spaces for engaging in discussions (Chaudhary et al. 2017, 37). Such interventions are key to combatting menstrual stigma, which remains prevalent. One woman explained, '[m]y brother asked me if there was a problem as I was staying inside for a long time. I just couldn't say out loud that I started my periods. I stayed inside the tent...' (Budhathoki et al. 2018, 4).

Most organizations paid little attention to the needs of particular groups, such as persons living with disabilities (Myers et al. 2018, 2-9). Dalit communities were marginalized by authorities and had particularly low access to services (Dalit Civil Society Massive Earthquake Victim Support and Coordination Committee 2015, 3, 34), demonstrating how caste-based discrimination is perpetuated in times of disasters and puts already marginalized individuals at even greater disadvantages.

This large-scale, sudden onset disaster poignantly demonstrates some of the issues faced by aid workers in administering menstrual health programming in the initial stages of an emergency: the enormous magnitude of the problem, the need to identify priorities of the affected population, and assumptions about what is 'culturally acceptable,' within a short time frame.

\section{Uganda}

Refugees in Uganda number around 1 million, with many settlements having existed for at least a decade (UNHCR 2018a). Uganda has adopted an approach which is more sustainable than in many other countries, giving refugees more freedom to develop livelihoods. For example, income, and imposes fewer restrictions on travel outside the settlements. However, a large part of the cost is borne by international donors (UNHCR 2018a).

Women and girls in Uganda in general face significant stress in dealing with menstruation due to taboos, feelings of shame, and unavailability of products (Miiro et al. 2018, 1). Presumably such shame exists not only in the minds of menstruators, but also in the minds of those around them (Miiro et al. 2018, 5-7). Therefore, WoMena has adopted an approach based on the Ecological Model, which goes from the individual level to the societal 
(Dahlberg and Krug 2002). The approach generally takes around six months to implement, and starts with the first point of contact with a community to assess whether there is interest in engaging with the program. If the community agrees to participate, next steps include training trainers and local supporters, including self-assigned 'role model men,' helping menstruators develop their skills and knowledge in using materials over a minimum of three months, then performing end-line surveys and consultations, and conducting follow-up after some months or years. Within this overall approach, WoMena frequently tries out small-scale variations as inspired by community suggestions (WoMena n.d.). For example, in the Bidibidi settlement pictorial training tools were used alongside relevant translations of the materials (WoMena 2019b; Elrha 2018). WoMena provides information on a wide range of menstrual materials, but solely distributes reusable materials, particularly menstrual cups and reusable pads. Cups are becoming increasingly popular in high income countries, but also in many countries in Africa, due to their low cost, high comfort, and sustainability. Reusable pads (particularly AfriPads) are provided as an alternative option to cups in many cases, as they have many of the same qualities (although they are less used in High Income Countries).

\section{Menstrual Shame and Stigma}

As mentioned above, in Uganda there is much shame, misinformation, and fear surrounding menstruation. Not surprisingly, this also seems to hold true for Uganda's refugee communities. Menstruators feel particular shame in washing and drying products. In Kitgum, for example, some women were ashamed that the menstrual blood stains on their cloth pads would be visible to others (Tellier, Hyttel, and Gad 2012, 8).

In many places around the world, parents, teachers, and girls see low understanding of menstruation as a problem, but are often too shy to address it. However, girls indicate that they are less shy to ask their parents about menstruation than about other issues related to SRHR (Ivanova et al. 2019, 1). WoMena's training approach and experience is that focusing on puberty education can be an entry point for sparking wider discussions on reproductive health (CARE International and WoMena Uganda 2018, 7), and for helping families and communities to become more open and less shameful. By the end of the Imvepi pilot, 94\% of girls had discussed menstruation with family members and friends after the project, whereas before it was considered taboo.

Community, especially male, involvement is also key to addressing stigma. Men may have concerns about menstrual irregularities caused by contraception (Hyttel et al. 2012, 151) and are often curious to learn more about menstruation, particularly because they are often the ones who pay for materials (CARE International and WoMena Uganda 2018, 6). One role model man from Imvepi stated, "I have understood that this (menstruation) is not a disease. Before I used to see it as something very bad, but nowadays, no problem" (CARE International and WoMena Uganda 2018, 7). 
Perhaps most importantly, WoMena's community-based model has helped to address the implications of stigma-induced stress, including heightened fear, as well as lack of agency and mobility (Tellier, Hyttel, and Gad 2012, 11). One of the most frequent reports by menstruators who have participated in WoMena supported activities is that they have gone from a state of fear to one of freedom. One beneficiary reported, "I feel free . . . Now I can drive my bike (to the food distribution site) and I don't have to look back" (Tellier, Hyttel, and Gad 2012, 6). These outcomes evidence the power that positive social attitudes toward menstruation have in improving menstruators' access to humanitarian programming.

\section{Health, Including Sexual and Reproductive Health and Rights (SRHR)}

One of the biggest concerns of menstruators relates to menstrual problems including cycle irregularities and pain management. In one study, only around a third of adolescents had ever in their lives consulted a medical doctor, with issues related to HIV and menstrual problems being the top reasons for seeking a consultation (Ivanova et al. 2019, 1). In response, WoMena has included information about such problems in educational material, including our FAQs explaining what is 'normal,' and when to see a health provider (WoMena 2018, 1-5). The training and kits have been adjusted to include more information and guidance on pain alleviation (for example, using a towel and bottle for warm compresses).

Menstrual health-related interpersonal violence is another significant concern and can take many forms. CARE reports that, in some cases, women were beaten by their husbands because they cut up distributed blankets to make pads (CARE International and WoMena Uganda 2018, 4). There are repeated reports by parents that puberty leads to early marriage, due not least to the fact that some cannot afford to buy pads for their daughters (CARE International and WoMena Uganda 2018,4). Girls report engaging in transactional sex to buy pads, for example Rachel (a Kitgum resident) says, "it (the project) should be extended to younger girls in school . . . a young girl can even get HIV-positive by looking for money to buy pads, if she prostitutes herself" (Tellier, Hyttel, and Gad 2012, 7). This demonstrates just some of the ways in which threats to physical well-being and inadequate health and management often go hand-in-hand.

\section{Access to Menstrual Materials, Including Affordability and Acceptability}

Affordability of menstrual materials is an overwhelming obstacle in Ugandan settlements once kits are no longer distributed. According to one trainer, "[s] ome women will sell their (food) rations but you can only get about [4 USD] from that. You then spend [1.6 USD] of that on pads alone and now you only have [2.4 USD] left" (CARE International and WoMena Uganda 2018, 24). This is confirmed by a mother in Rhino settlement, who shared that, "when you arrive in the camp they give you three packets of pads which each 
packet contains 10 pieces but if it gets over and we don't receive other pads we use piece of clothes [sic]" (Norelius 2017, 47).

To save money, menstruators report resorting to changing disposable pads less often than suggested by manufacturer guidelines (Norelius 2017, 47 ), sharing with others, or washing and reusing disposable pads (WoMena 2019a, 1). Disposable pads cost 18-35 USD per year, reusable pad kits such as AfriPads cost around 5 USD per kit and last $1-2$ years, while cups sell at 5-15 USD and last up to 10 years (Tellier and Hyttel 2018, 20). Pilot projects supported by WoMena and its partners indicate that menstruators are willing to pay 4-7 USD for a cup (Tellier and Hyttel 2018, 29).

At the same time, acceptability of products is equally important. There are often questions and concerns (which are at times posed by humanitarian staff rather than menstruators) regarding whether menstrual cups or other reusable materials are 'culturally acceptable': Will it affect the hymen? Will it hurt? Will it be difficult to use? These legitimate concerns must be taken seriously, and both the negative and positive potential results of each product choice should be openly considered.

The end-line study from Kitgum indicates that the overall experience for the participants using cups was either very good $(75 \%, n=18)$ or $\operatorname{good}(25 \%$, $n=6$ ) (Tellier, Hyttel, and Gad 2012, 2). The pilot project in Rhino settlement indicated that initial uptake was higher for reusable pads than cups (100\% versus 69\%). Consistent use was slightly higher for cups than reusable pads $(87 \%$ versus $74 \%)$, as users overcame initial difficulties. Beneficiary satisfaction rates were high for both materials, and increased over time as menstruators got accustomed to each. The highest satisfaction rates were found among beneficiaries who were provided both materials. They could alternate, for example, using cups when there was insufficient water or on heavy flow days (Gade and Hytti 2017, 8, 32). The presence of experienced users who could answer questions was key in continued use, especially cups. Kits and training were also modified to meet concerns. For example, in response to some experiencing challenges boiling the cup, a metal container for boiling was added to the kit (Tellier, Hyttel, and Gad 2012, 8).

Regarding the quality and acceptability of kits, it is also important to ask: Acceptable to whom? At what time? How (for example, what type of interaction with the community)? WoMena has found that refugee populations may be more open to changing their practices, and possess more of the time needed to engage in consultations, than populations living in more stable conditions. While this is only a formative impression, it has been confirmed by others (Sphere Association 2018, 75; VanLeeuwen and Torondel 2018 , 355). Outside of menstruators' personal preferences, as well as the quality and contextual acceptability of menstrual products, practicalities like those concerning water, sanitation, and hygiene must also be taken into consideration. 


\section{Water, Sanitation, Hygiene, Including Environmental Concerns}

One estimate from the Imvepi project is that it takes each menstruator around 15 liters per cycle to wash reusable products, including hands and soiled underwear. Here, cups have an advantage, since they can be used without underwear, need an estimated 1-2 liters per cycle, and do not need to be dried. The main complication is that they need to be boiled once a month (Hyttel et al. 2017). One Imvepi participant noted she was happy to use a cup, "because it uses less water and [her] grandmother supports [her] in using it because of that" (CARE International and WoMena Uganda 2018, $10)$.

\section{Populations with Particular Needs}

WoMena's consultative approach helps to identify menstruators with special needs. This includes a wide range of disabilities which must be taken into account, as menstruators with limited mobility (for example, if they have lost a leg) or cognitive disabilities, as well as their caregivers, have particular needs. This is an area where a systematic response might be further developed (WoMena n.d.).

In sum, long-term emergencies present very different needs than acute situations. WoMena's experiences in the Ugandan context suggest refugees may be relatively open to trying out innovative approaches. Working with a more developmental approach and engaging widely with communities not only seems to afford greater possibilities of overcoming the practical issues related to materials, water, and sanitation, but also presents opportunities to change social attitudes toward menstruation.

\section{CONCLUSION}

Over the last few decades there has been increased attention to menstruation in humanitarian settings. The reproductive health sector has been a relative newcomer to this discussion, but even at this early stage of engagement, we find that discussions on menstrual health are proving to be a potent entry point for reducing shame and ignorance about a host of sexual and reproductive health and rights.

Challenges remain to achieving standardized, coordinated, and sustainable programming, namely in: reaching policy agreements on what the basic contents of emergency response should be, and determining who would be responsible for different tasks at different stages of response. The intention should be that, over time, a transition can be made from a narrow humanitarian 'three-month kit' culture to one of the more sustainable solutions. What is more, determining what is 'culturally acceptable' plays a key role in developing such solutions. Culture is a fluid concept, and 'acceptability' needs to address questions such as: acceptable to whom? when? and how? WoMena's experience is that refugees may be quite open to trying out innovative approaches, and have more time to do so, than people in stable settings. After 
all, they have already demonstrated their adaptability and agency by fleeing and adjusting to new living conditions.

In a broader perspective, the transition from dealing with acute emergencies to a longer term, sustainable approach is a high profile and persistent challenge for the entire humanitarian field. At times, these challenges are partially presented by the competing approaches utilized by actors from different fields. In humanitarian assistance, 'neutrality' is traditionally a key concept (ICRC 1979, 7), meaning that one may need to work around local institutions, to ensure impartiality in administering aid. Meanwhile, in development assistance, one central principle is usually 'participation,' which includes working through local institutions to build long-term solutions, after the initial stages of an emergency have passed (UNDG 2003).

Finally, context matters. In humanitarian action, the approach is often to have a blueprint for the first few months, with the intention that the approach should be 'contextualized' in the long term. Uganda hosts one of the largest long-term refugee populations in sub-Saharan Africa, and employs more sustainable approaches to providing aid than many other countries in the world (UNHCR 2016). This can help create an environment for a developmental approach that is tailored to the needs of menstruators. In conclusion, comparing the situations in Nepal and Uganda may help in finding some small-scale, practical example of how to bridge the gap between short- and longer-term approaches in humanitarian assistance.

\section{Notes}

1. We would like to acknowledge the excellent research assistance provided by Sydney Amoakoh.

2. An internationally recognized set of common principles and universal minimum standards in humanitarian response. Source: https://www.spherestandards.org/ about.

\section{REFERENCES}

Abbott, L., B. Bailey, Y. Karasawa, D. Louis, S. McNab, D. Patel, C. Lopez, R. Rani, C. Saba, and L. Vaval. 2011. Evaluation of UNFPA's Provision of Dignity Kits in Humanitarian and Post-Crisis Settings. School of International and Public Affairs, Columbia University.

ACF, UNHCR, and UNICEF. 2017. Results from a Pilot with the Disinfecting Menstrual Hygiene Pad 'Safepad ${ }^{\mathrm{TM}}$ ' Azraq Refugee Camp-Jordan.

Atuyambe, Lynn M., Michael Ediau, Christopher G. Orach, Monica Musenero, and William Bazeyo. 2011. "Landslide Disaster in Eastern Uganda: Rapid Assessment of Water, Sanitation and Hygiene Situation in Bulucheke Camp, Bududa District." Environmental Health 10 (1): 38.

Balls, Emily, Robert Dreiselbis, and Belen Torondel. 2017. Menstrual Hygiene Management: Policy Brief. London: SHARE Consortium. http://www.susana. org/en/resources/library/details/2787. 
Blanchet, K., A. Ramesh, et al. 2017. "Evidence on Public Health Interventions in Humanitarian Crises." Lancet 390 (10109): 2287-96.

Budhathoki, Shyam Sundar, Meika Bhattachan, Enrique Castro-Sánchez, Reshu Agrawal Sagtani, Rajan Bikram Rayamajhi, Pramila Rai, and Gaurav Sharma. 2018. "Menstrual Hygiene Management among Women and Adolescent Girls in the Aftermath of the Earthquake in Nepal.” BMC Women's Health 18 (1): 33.

CARE International and WoMena Uganda. 2018. Ruby Cups: Girls in Imvepi Refugee Settlement Taking Control.

Chaudhary, Pushpa, Giulia Vallese, Meera Thapa, Valerie Broch Alvarez, Latika Maskey Pradhan, Kiran Bajracharya, Kazutaka Sekine, Shilu Adhikari, Reuben Samuel, and Sophie Goyet. 2017. "Humanitarian Response to Reproductive and Sexual Health Needs in a Disaster: The Nepal Earthquake 2015 Case Study." Reproductive Health Matters 25 (51): 25-39.

Chynoweth, S. K. 2015. "Reproductive Health in the Changing Humanitarian Context-Findings from the IAWG on Reproductive Health in Crises' 2012-14 Global Evaluation." Conflict and Health 9: 1-154.

Dahlberg, L. L., and E. G. Krug. 2002. "Violence-A Global Public Health Problem." In World Report on Violence and Health, edited by E. Krug, L. L. Dahlberg, J. A. Mercy, A. B. Zwi, and R. Lozano. Geneva, Switzerland: World Health Organization.

Dalit Civil Society Massive Earthquake Victim Support and Coordination Committee. 2015. Waiting for "Justice in Response": Report of Initial Findings from Immediate Needs Assessment and Monitoring Responses towards Affected Dalit Communities in Nepal Earthquake 2015.

Department of Women, South Africa, and UNFPA. 2018. First East and Southern Africa Regional Symposium Menstrual Health Management for Adolescent Girls and Women. Johannesburg. https://esaro.unfpa.org/sites/default/files/pub-pdf/ MHM\%20Report\%20final\%20digital.pdf.

Elrha. 2018. "Ndrelmba, Perotiyapa and Dora: Translating Menstruation." Accessed February 26, 2019. https://www.elrha.org/project-blog/ndrelmba-perotiyapaand-dora-translating-menstruation/.

Elledge, Myles, Arundati Muralidharan, Alison Parker, Kristin Ravndal, Mariam Siddiqui, Anju Toolaram, and Katherine Woodward. 2018. "Menstrual Hygiene Management and Waste Disposal in Low and Middle Income Countries-A Review of the Literature." International Journal of Environmental Research and Public Health 15 (11): 2562.

Gade, Anna, and Laura Hytti. 2017. Menstrual Health in Rhino Camp Refugee Settlement, West Nile, Uganda, Pilot Project Intervention Report. Kampala: WoMena Uganda and ZOA.

Hyttel, Maria, Jennifer J. K. Rasanathan, Marianne Tellier, and Willington Taremwa. 2012. "Use of Injectable Hormonal Contraceptives: Diverging Perspectives of Women and Men, Service Providers and Policymakers in Uganda." Reproductive Health Matters 20 (40): 148-57.

Hyttel, Maria, Camilla Faldt Thomsen, Bianca Luff, Halvor Storrusten, Viola Nilah Nyakato, and Marianne Tellier. 2017. "Drivers and Challenges to Use of Menstrual Cups among Schoolgirls in Rural Uganda: A Qualitative Study.” Waterlines 36 (2): $109-24$.

International Committee of the Red Cross (ICRC). 1979. The Fundamental Principles of the Red Cross: commentary. 
Ivanova, Olena, Masna Rai, Wendo Mlahagwa, Jackline Tumuhairwe, Abhishek Bakuli, Viola N. Nyakato, and Elizabeth Kemigisha. 2019. "A Cross-Sectional Mixed-Methods Study of Sexual and Reproductive Health Knowledge, Experiences and Access to Services among Refugee Adolescent Girls in the Nakivale Refugee Settlement, Uganda." Reproductive Health 16 (1): 35. https://doi.org/10.1186/ s12978-019-0698-5.

Johnston-Robledo, Ingrid, and Joan C. Chrisler. 2013. "The Menstrual Mark: Menstruation as Social Stigma." Sex Roles 68 (1-2): 9-18. https://link.springer. com/article/10.1007/s11199-011-0052-z.

Kågesten, Anna E., Linnea Zimmerman, Courtland Robinson, Catherine Lee, Tenaw Bawoke, Shahd Osman, and Jennifer Schlecht. 2017. "Transitions Into Puberty and Access to Sexual and Reproductive Health Information in Two Humanitarian Settings: A Cross-Sectional Survey of Very Young Adolescents From Somalia and Myanmar." Conflict and Health 11 (1): 24.

Kragelund Nielsen, Johanne. 2017. "Women and Girls' Experiences with Safety, Hygiene and Sanitation in Connection with Menstrual Health Management in Nyarugusu Refugee Camp.” Master's thesis, University of Copenhagen.

Miiro, George, Rwamahe Rutakumwa, Jessica Nakiyingi-Miiro, Kevin Nakuya, Saidat Musoke, Juliet Namakula, Suzanna Francis, et al. 2018. "Menstrual Health and School Absenteeism among Adolescent Girls in Uganda (MENISCUS): A Feasibility Study." BMC Women's Health 18 (1): 4.

Myers, Anna, Samira Sami, Monica Adhiambo Onyango, Hari Karki, Rosilawati Anggraini, and Sandra Krause. 2018. "Facilitators and Barriers in Implementing the Minimum Initial Services Package (MISP) for Reproductive Health in Nepal Post-Earthquake." Conflict and Health 12 (1): 1-9.

Norelius, Hanna Maria. 2017. "Managing Menstruation During Displacement: A Mixed Methods Study Investigating Menstrual Hygiene Management in Rhino Refugee Settlement. Uganda.” Master's thesis, University of Copenhagen.

Parker, Alison H., Jen A. Smith, Tania Verdemato, Jeanette Cooke, James Webster, and Richard C. Carter. 2014. "Menstrual Management: A Neglected Aspect of Hygiene Interventions." Disaster Prevention and Management 23 (4): 437-54.

Phillips-Howard, Penelope A., Elizabeth Nyothach, Feiko O. ter Kuile, Jackton Omoto, Duolao Wang, Clement Zeh, Clayton Onyango, et al. 2016. "Menstrual Cups and Sanitary Pads to Reduce School Attrition, and Sexually Transmitted and Reproductive Tract Infections: A Cluster Randomised Controlled Feasibility Study in Rural Western Kenya." BMJ Open 6 (11): 1-11.

Pujol-Mazzini, Anna. 2017. "For Refugee Women, Periods a Dangerous, Shameful Time." Reuters, March 8. https://www.reuters.com/article/us-womens-day-refugees-periods-feature/for-refugee-women-periods-a-dangerous-shameful-timeidUSKBN16F1UU.

Rasanathan Jennifer, J. K., Andisheh Jahangir, Mary Grace Sandy, Marianne Tellier, and Siri Tellier (WoMena). 2019. Doubly Left Behind at the Intersection of Disability and Menstruation. Manuscript submitted for publication.

Robinson, Alice, and Alice Obrecht. 2016. "Improving Menstrual Hygiene Management in Emergencies: IFRC's MHM Kit." HIF/ALNAP Case Study. London: ODI/ ALNAP.

Samari, Goleen. 2017. "Syrian Refugee Women's Health in Lebanon, Turkey, and Jordan and Recommendations for Improved Practice." World Medical \& Health Policy 9 (2): 255-74. 
Sohrabizadeh, Sanaz, Katayoun Jahangiri, and Reza Khani Jazani. 2018. "Reproductive Health in the Recent Disasters of Iran: a Management Perspective." BMC Public Health 18 (1): 389.

Sommer, Marni. 2012. "Menstrual Hygiene Management in Humanitarian Emergencies: Gaps and Recommendations." Waterlines 31 (1-2): 83-104.

Sommer, Marni, Margaret L. Schmitt, David Clatworthy, Gina, Bramucci, Erin Wheeler, and Ruwan Ratnayake. 2016. "What Is the Scope for Addressing Menstrual Hygiene Management in Complex Humanitarian Emergencies? A Global Review." Waterlines, Practical Action Publishing 35 (3): 245-64. https:// doi.org/10.3362/1756-3488.2016.024.

Sommer, M., M. Schmitt, and D. Clatworthy. 2017. A Toolkit for Integrating Menstrual Hygiene Management (MHM) into Humanitarian Response. New York: Columbia University, Mailman School of Public Health and International Rescue Committee.

Sphere Association. 2018. The Sphere Handbook: Humanitarian Charter and Minimum Standards in Humanitarian Response. 4th ed. Geneva: Sphere Association. www. spherestandards.org/handbook.

Tellier, M., M. Hyttel, and M. Gad. 2012. Assessing Acceptability and Hygienic Safety of Menstrual Cups as Menstrual Management Methods for Vulnerable Young Women in Uganda Red Cross Society's Life Planning Skills Project. Kampala: WoMena Ltd.

Tellier, Siri, and Maria Hyttel (WoMena). 2018. "Menstrual Health Management in East and Southern Africa: A Review Paper.” Johannesburg: UNFPA.

Tellier, Siri, Andreas Kiaby, Lars Peter Nissen, Jonas Torp Ohlsen, Wilma Doedens, Kevin Davies, Dan Brun Petersen, Vibeke Brix Christensen, and Niall Roche. 2017. "Basic Concepts and Current Challenges of Public Health in Humanitarian Action.” In International Humanitarian Action, 229-317. Cham: Springer.

Torondel, Belen, Shalini Sinha, Jyoti Ranjan Mohanty, Tapoja Swain, Pranati Sahoo, Bijaya Panda, Arati Nayak, et al. 2018. "Association between Unhygienic Menstrual Management Practices and Prevalence of Lower Reproductive Tract Infections: A Hospital-Based Cross-Sectional Study in Odisha, India." BMC Infectious Diseases 18 (1): 1-12. https://doi.org/10.1186/s12879-018-3384-2.

UN General Assembly. 1992. Strengthening of the Coordination of Humanitarian Emergency Assistance of the United Nations: Resolution, Adopted by the General Assembly, April 14, 1992, A/RES/46/182. https://www.refworld.org/ docid/3b00fl8620.html.

UN High Commissioner for Refugees (UNHCR). 2016. "Global Trends: Forced Displacement in 2015." June 20. Accessed September 14, 2018. http://www.refworld.org/docid/57678f3d4.html.

UN High Commissioner for Refugees (UNHCR). 2018a. "Global Focus-Uganda." Accessed January 7, 2019. http://reporting.unhcr.org/node/5129.

. 2018b. "Global Trends-Forced Displacement in 2017." Accessed September 14, 2018. http://www.unhcr.org/globaltrends2017/.

UN Sustainable Development Group (UNDG). 2003. “The Human Rights Based Approach to Development Cooperation towards a Common Understanding among UN Agencies." May 3-5.

VanLeeuwen, Crystal, and Belen Torondel. 2018. "Improving Menstrual Hygiene Management in Emergency Contexts: Literature Review of Current Perspectives." International Journal of Women's Health 10: 169-86. https://doi.org/10.2147/ IJWH.S135587. 
World Health Organization (WHO). 1946. Preamble to the Constitution of the World Health Organization as Adopted by the International Health Conference, New York, June 19-22 (Official Records of the World Health Organization, no. 2, p. 100).

World Health Organization (WHO), and the United Nations International Children's Emergency Fund (UNICEF). 2012. Report of the Second Consultation on Post-2015 Monitoring of Drinking-Water, Sanitation and Hygiene, WHO/ UNICEF Joint Monitoring Programme for Water Supply and Sanitation (JMP), Hosted by the Royal Government of the Netherlands in the Hague, December 3-5, 2012: 89.

WoMena. 2018. "WoMena FAQs: Menstrual Irregularities." http://womena.dk/ womena-faqs-what-are-menstrual-irregularities/.

WoMena. 2019a. "WoMena FAQs: Is There Any Connection between Menstrual Cups and Infections?" http://womena.dk/is-there-any-connection-between-menstrualcups-and-infections/.

WoMena. 2019b. "Photo Story: Meeting Linguistic Challenges with Image." http:// womena.dk/photo-story-meeting-linguistic-challenges-with-images/.

WoMena. n.d. Programmatic Approach. Accessed September 19, 2018. http://womena.dk/goals-strategies-policies/.

Women's Refugee Commission. 2016. Mean Streets: Identifying and Responding to Urban Refugees' Risks of Gender-Based Violence. https://www.womensrefugeecommission.org/images/zdocs/mean-streets-urban-gbv-sex-workers.pdf.

Open Access This chapter is licensed under the terms of the Creative Commons Attribution 4.0 International License (http://creativecommons.org/licenses/ by $/ 4.0 /$ ), which permits use, sharing, adaptation, distribution and reproduction in any medium or format, as long as you give appropriate credit to the original author(s) and the source, provide a link to the Creative Commons license and indicate if changes were made.

The images or other third party material in this chapter are included in the chapter's Creative Commons license, unless indicated otherwise in a credit line to the material. If material is not included in the chapter's Creative Commons license and your intended use is not permitted by statutory regulation or exceeds the permitted use, you will need to obtain permission directly from the copyright holder.

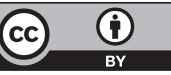

